Boletín de la Sociedad Zoológica del Uruguay, 2020

Vol. 29 (2): 160-166

ISSN 2393-6940

https://journal.szu.org.uy

\title{
RELEVAMIENTO DE ARAÑAS EN LA UNIDAD DE INTERNACIÓN DE PERSONAS PRIVADAS DE LIBERTAD NÚMERO 6 "PUNTA DE RIELES": UNA EXPERIENCIA EDUCATIVA
}

\section{Bardier Gustavo1, Mereles Luis ${ }^{1}$, Costabel Norberto1, Vezzoli Sebastián1, Perlipe Andrés ${ }^{1}$, López Daniel1$^{1}$, Parodi Gerardo1, Tambasco Ramiro ${ }^{2-4}$, Rojas-Buffet Carolina ${ }^{2,3,4}$ \& Viera Carmen²,4}

1. Unidad de Internación de Personas Privadas de Libertad № 6 "Punta de Rieles" Instituto Nacional de Rehabilitación, Ministerio del Interior, Uruguay.

2. Departamento de Biología Animal, Sección Entomología, Facultad de Ciencias, Universidad de la República, Uruguay.

3. Departamento de Ecología y Biología Evolutiva, Instituto de Investigaciones Biológicas Clemente Estable, Ministerio de Educación y Cultura, Uruguay.

4. Laboratorio Ecología del Comportamiento, Instituto de Investigaciones Biológicas Clemente Estable, Ministerio de Educación y Cultura, Uruguay.

Autor para correspondencia: cviera@ fcien.edu.uy

\section{RESUMEN}

La experiencia educativa y de investigación se realizó en contexto de encierro con seis personas privadas de libertad y un monitor en territorio. Se relevaron arañas en tres sitios de la Unidad penitenciaria: alojamiento de privados de libertad, campo en abandono y huerta orgánica. Se manejó la hipótesis de que la diversidad se vería influenciada por disturbios ambientales producidos por humanos. Los participantes fueron formados en los conceptos básicos del método científico, aplicado al estudio de la diversidad y composición de la araneofauna local. Se realizaron muestreos estacionales durante un año, se recolectó y acondicionó el material. Se identificaron 682 arañas pertenecientes a 18 familias. Se encontró mayor riqueza en campo en abandono y menor en vivienda, por tanto, se corroboró la hipótesis. En verano se encontró la mayor abundancia de arañas. Las familias más representadas fueron Lycosidae y Linyphiidae. El número de familias por estación fue similar, aunque varió en su composición. Se encontró actividad reproductiva en seis familias, la mayoría en primavera y verano. Este trabajo permitió un rico intercambio y fue un desafío educativo, debido a las restricciones de infraestructura y materiales permitidos en la unidad penitenciaria. Se generó el trabajo en un clima de respeto y responsabilidad.

Palabras clave: Araneofauna, Relevamiento, Educación, Confinamiento.

* Proyecto de investigación financiado por la Comisión de Educación Permanente de la Universidad de la República.

* Research project financed by the Commission of Permanent Education of the University of the Republic.

\section{ABSTRACT}

Spider sampling at the unit of persons private of liberty number 6 "Punta de Rieles": An educative experience. The educational and research experience was carried out in the context of confinement with six people deprived of liberty and a monitor in the territory. Spiders were sampled in three sites of the Penitentiary Institution: rooms of Persons Deprived of Liberty, wild vegetation field and organic crops. The hypothesis was that diversity would be influenced by environmental disturbances produced by humans. The participants received information about the animal group to analyze diversity and composition of the spider fauna. Seasonal samplings were carried out for a year, the material was collected and conditioned. There were 682 spiders belonging to 18 families. Higher richness was found in abandoned fields and lower in housing, therefore, the hypothesis was corroborated. In summer the greatest abundance of spiders was found. The most represented families were Lycosidae and Linyphiidae. The number of families per season was similar. Reproductive activity was found in six families, the majority in spring and summer. This work allowed a rich exchange and was an educational challenge, due to the infrastructure and material restrictions allowed in the Penitentiary Institution. The study was generated in a climate of respect and responsibility.

Keywords: Spider fauna, Sampling, Education, Confinement.

\section{INTRODUCCIÓN}

El presente trabajo se realizó en el marco de los cursos y talleres de Educación Permanente de la Facultad de Ciencias, UdelaR. Para ello, se realizó un proyecto entre dos docentes e investigadores y un Monitor profesional. Para acercarnos a la actualización del conocimiento de nuestra fauna, utilizamos como modelo animal a las arañas y su 
papel en los ecosistemas terrestres. Para ello diseñamos un relevamiento araneológico en tres sitios diferentes de la Unidad penitenciaria y se comparó la presencia diferencial del grupo a nivel taxonómico de familias.

La Unidad № 6 Punta de Rieles de Montevideo es un centro penitenciario modelo, con algunas peculiaridades que nos permitieron desarrollar la actividad con ciertas facilidades, pese al contexto de encierro. Allí se trabaja con un sistema semiabierto, donde unas 600 personas privadas de libertad pueden transitar por toda la cárcel desde las 8 hasta las 18 hs. Durante ese tiempo, realizan múltiples tareas laborales, educativas u de recreación. Se han organizado pequeñas empresas autogestionadas para sobrevivir y acceder al consumo interno, por ejemplo: almacenes, peluquerías, panaderías, bloqueras, entre otras. Además, participan de grupos de teatro, de una radio comunitaria, desarrollan deportes al aire libre en canchas para ese propósito, disponen de un gimnasio cerrado y de un anfiteatro que se usa para talleres, cine, exposiciones, etc. Algunas de las personas continúan con sus estudios curriculares de escuela, liceo y Universidad. El monitor del equipo de campo, Gustavo Bardier, funcionario de la Unidad y Magister en Biología, fue estratégico para la organización de las tareas de relevamiento y el acondicionamiento del material recolectado, y además para facilitar el trabajo entre los estudiantes y los docentes de Facultad de Ciencias.

El Proyecto de Extensión y Convenio entre la dirección de la Unidad penitenciaria y el Decano de la Facultad de Ciencias se enmarca en una concepción universitaria acerca de hacer vivo el concepto de integralidad. Un desafío que presenta la integralidad es el formato en que se brinda regularmente la educación universitaria, de manera escolarizada y práctica de tipo aúlica (Arocena et al., 2011). Por ello, incursionamos con este Proyecto en la integración de los procesos de enseñanza, creación de conocimientos y extensión en un contexto de encierro. Se han realizado varios estudios de diversidad araneológica en nuestro país para conocer la araneofauna local en el marco del mantenimiento de la diversidad (Costa \& Simó, 2014; Simó et al., 2015; Laborda et al., 2018). Teniendo en cuenta la importancia de la conservación de la diversidad y el impacto de la actividad humana se propuso relevar la araneofauna en tres sitios con diferente intervención humana, con la hipótesis de que la diversidad sería más alta en sitios menos intervenidos. Torres et al. (2016) y Ruiz (2017) han utilizado a la araneofauna como grupo bioindicador de la calidad ambiental y efectos antrópicos, en contextos socioambientales diferentes. Esta experiencia científico-educativa es inédita en cárceles.

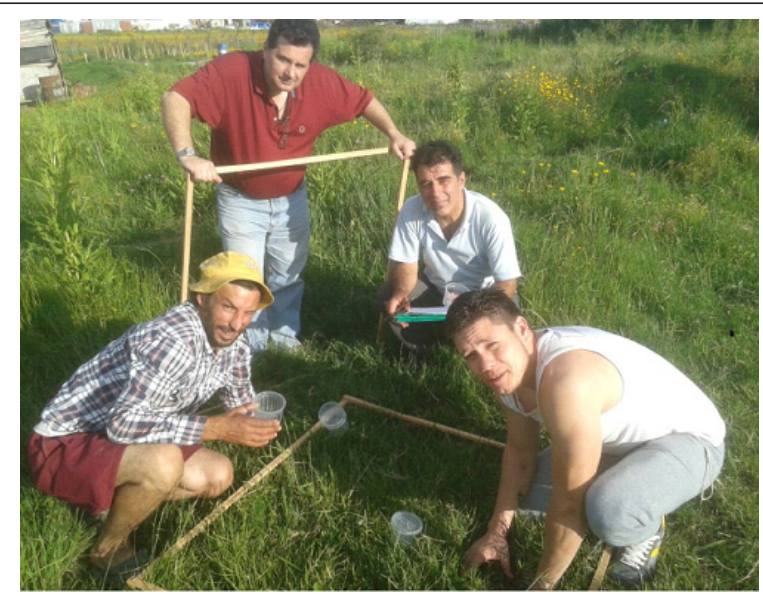

Fig. 1. Estudiantes junto al monitor realizando el relevamiento manual de arañas con cuadrantes en el campo en abandono.

\section{MATERIAL Y MÉTODOS}

El monitor reunió un grupo de trabajo con personas privadas de libertad (PPL) interesadas en el «mundo de los animales». Previo a la realización de los relevamientos, se brindaron dos talleres sobre biología de arácnidos, su biodiversidad en Uruguay y una introducción a los métodos de muestreo, y tres clases teóricas y prácticas sobre la metodología tendiente a sistematizar el relevamiento. Todas las actividades formativas teóricas y experimentales fueron realizadas en la Unidad de Punta de Rieles. También se trabajó el tema de arañas peligrosas, reconocimiento y necesidad de tomar precauciones, para el caso que se encontraran algunas durante el relevamiento. A cada participante se le obsequió el libro Arácnidos de Uruguay (Viera, 2011) y materiales para tomar apuntes. Dicho libro recopila el conocimiento sistemático de las arañas autóctonas y contiene claves para determinar las familias más representativas del país. También se brindó acceso a material bibliográfico relacionado con la determinación sistemática de los ejemplares recolectados (Benamú, 2007; Grismado et al., 2014) y aspectos de conservación y ecología (Viera \& Gonzaga, 2017).

En relación a los aspectos educativos, a seis personas privadas de libertad se les transmitieron los aspectos metodológicos y los pasos necesarios para la obtención de conocimiento original. Para ello, se trabajó desde el inicio estimulando la curiosidad de los estudiantes, planteando preguntas, elaborando hipótesis y colaborando en la estrategia metodológica a utilizar para la comprobación de estas hipótesis. 


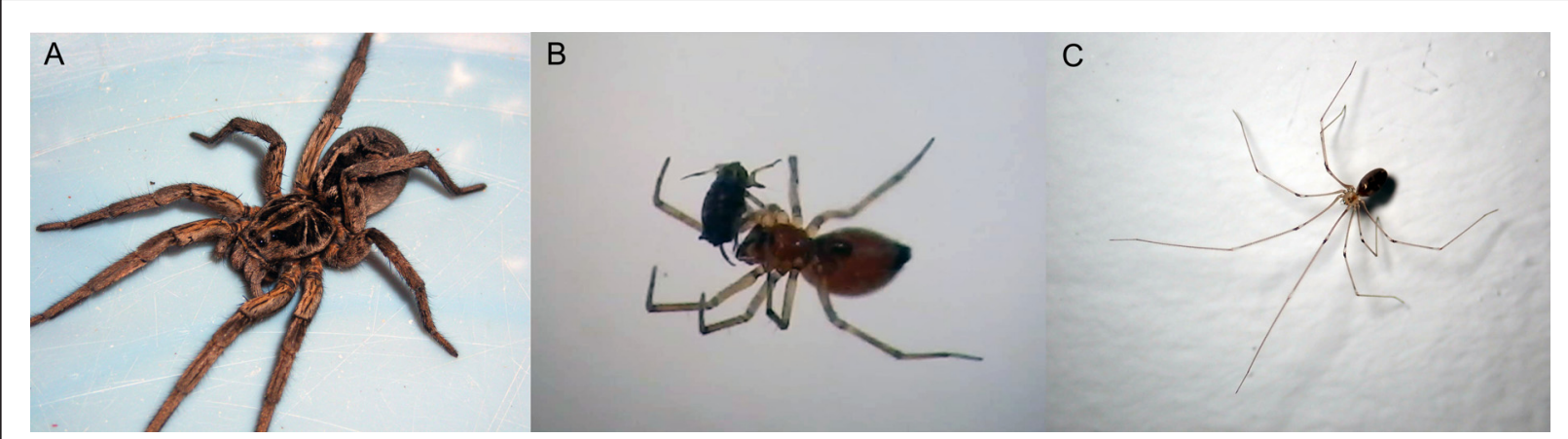

Fig. 2. Tres familias de arañas con mayor abundancia. A. Lycosidae. B. Linyphiidae. C. Pholcidae.

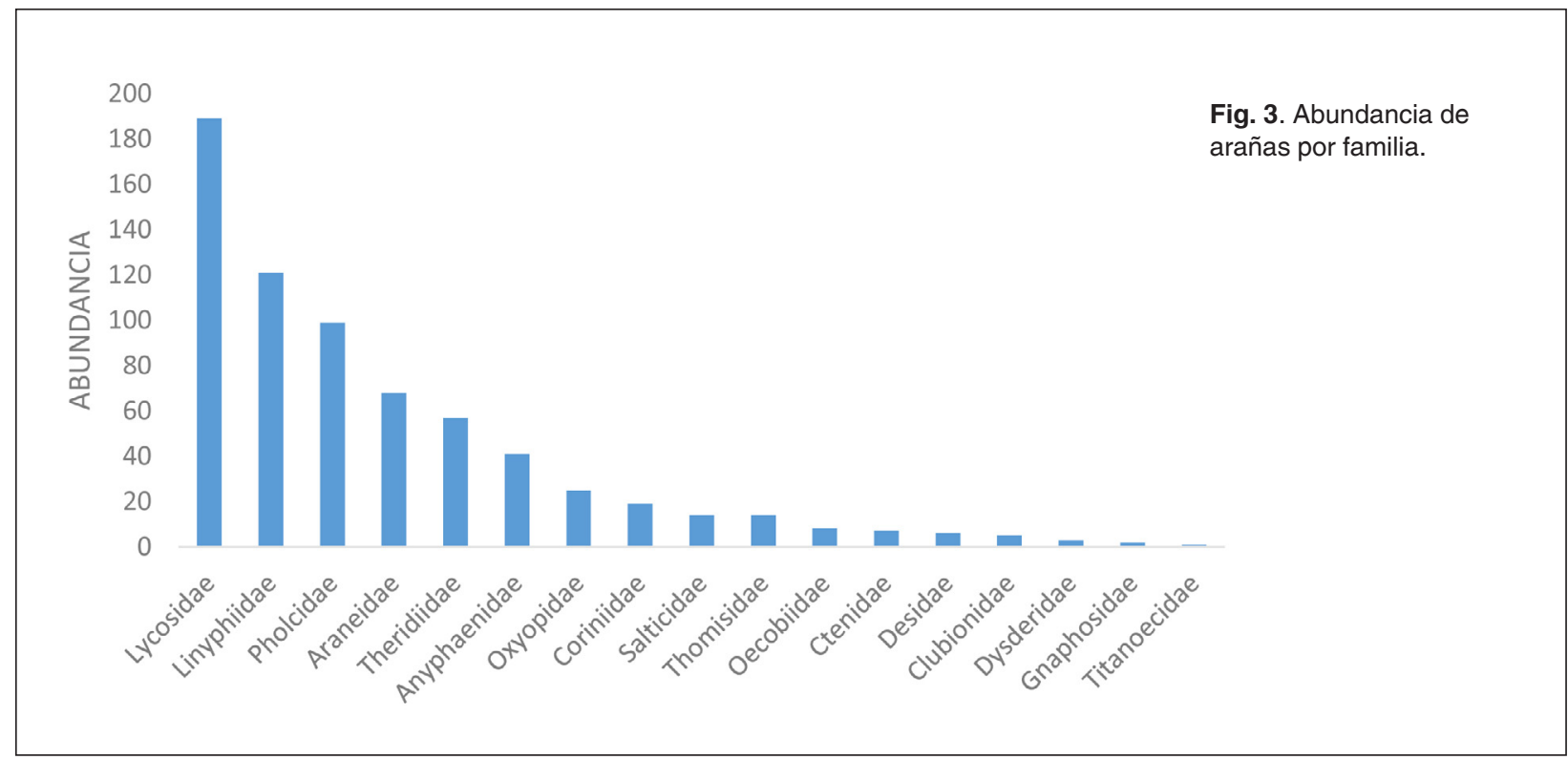

\section{Áreas de estudio}

El estudio se realizó en tres sitios: un campo con vegetación silvestre, con predominancia de pastizal bajo; una huerta orgánica de hortalizas y maíz; y un sector de alojamiento de los internos, que incluye habitaciones, pasillos, comedor, cocina y baños. Las áreas relevadas se escogieron teniendo en cuenta un posible gradiente de presencia humana: vivienda, con mayor influencia humana; huerta, con incidencia media y campo con vegetación silvestre con pastizales y chircas, con un mínimo de intervención humana. Los relevamientos fueron estacionales, a lo largo de un año, en la primavera de 2016, verano, otoño e invierno de 2017.

Los métodos de colecta variaron según los sitios. En la vivienda se recolectaron manualmente ad libitum, con un esfuerzo de colecta de tres colectores durante media hora. En la huerta y en el campo con vegetación silvestre, se utilizó el método del cuadrante (10 de 1 $\mathrm{m}^{2}$ ), distribuidos al azar, con un esfuerzo de colecta de dos colectores durante media hora por cuadrante (Fig. 1). Se registraron las arañas en actividad reproductora, teniendo en cuenta como indicador la presencia de ootecas y/o crías.

Debido a las restricciones de la Unidad (ingreso prohibido de objetos punzantes, alcohol y/o vidrio) fue necesario que los estudiantes fabricaran sus propios materiales de recolección. Estas tareas complementaron las habilidades y destrezas que se habían planteado en el proyecto. Las arañas recolectadas fueron acondicionadas en envases plásticos numerados. Los ejemplares fueron registrados en fichas de campo donde se constaba el número de individuo; fecha y hora de la recolección; humedad y temperatura del ambiente; sitio de colecta; método de colecta, y observaciones. Se identificó el sexo de los ejemplares, su estado de desarrollo y se determinaron a nivel de familia. Para el reconocimiento 


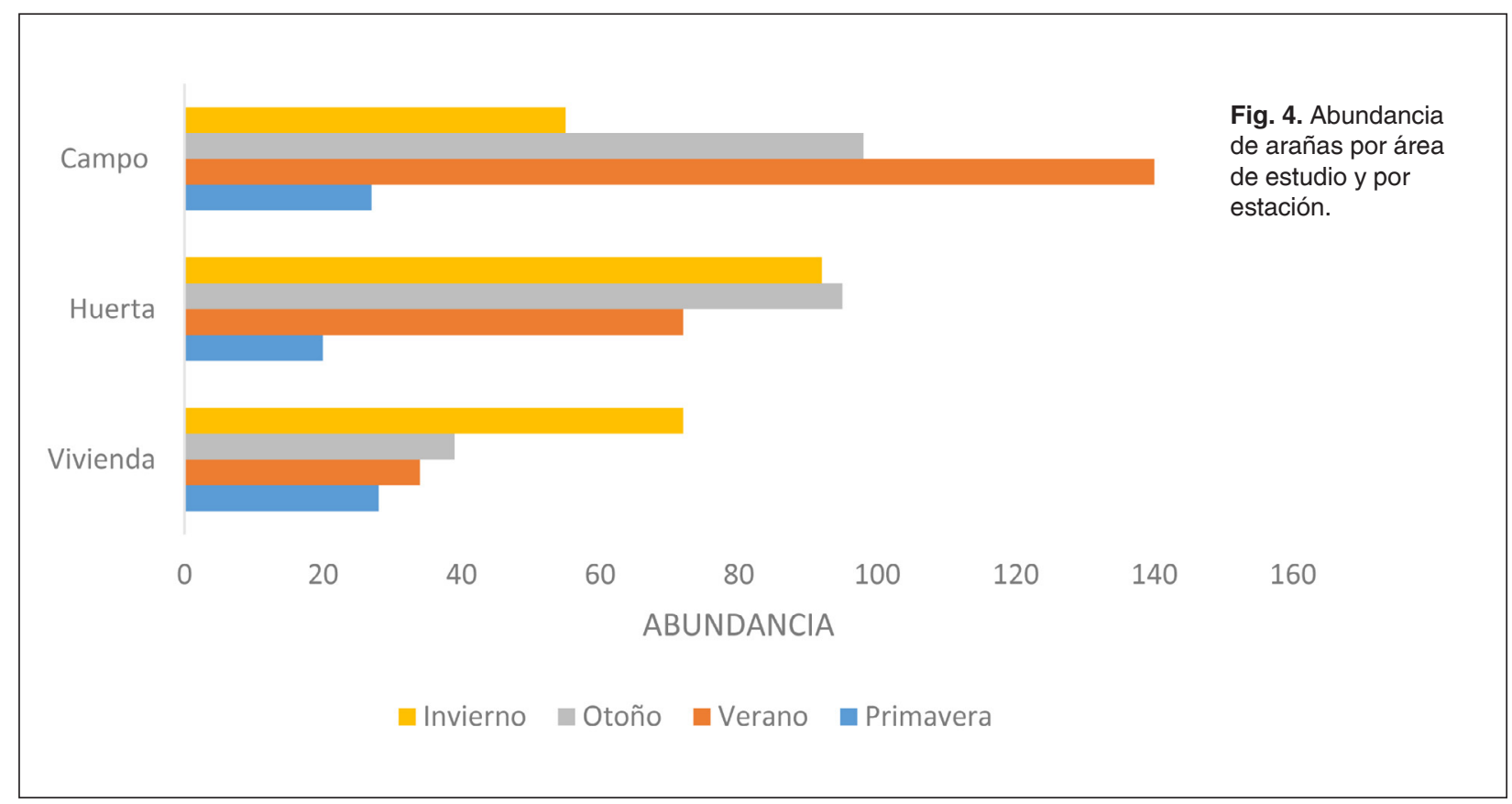

de las familias, se utilizaron como apoyo el libro Arácnidos de Uruguay (Viera, 2011), la guía de Arácnidos del Uruguay (Aisenberg et al, 2011) y las claves de las principales familias presentes en el país y la región (Benamú, 2007; Grismado et al., 2014). Durante la realización del relevamiento se mantuvo un contacto fluido con los estudiantes, presencial con el monitor y mediante consultas por mail con los investigadores. Las arañas que murieron en la Unidad fueron conservadas en agua y sal gruesa (debido a no tener permitido el uso de alcohol). La filtración de las muestras se realizó en el Penal y la determinación taxonómica se realizó por los investigadores en el Laboratorio de Ecología del Comportamiento del Instituto de Investigaciones Biológicas Clemente Estable y la Sección Entomología de la Facultad de Ciencias, mediante lupa binocular y las claves de determinación ya mencionadas. Se comparó la abundancia de arañas y la diversidad de familias, teniendo en cuenta los sitios de muestreo y las variaciones de acuerdo a las estaciones. Se analizaron las diferencias encontradas en los tres sitios, de acuerdo con lo usualmente utilizado (Viera \& Gonzaga, 2017).

\section{RESULTADOS}

\section{Relevamiento araneológico}

Se recolectaron un total de 772 arañas, 682 adultas, pertenecientes a 18 familias de arañas. Las familias con mayor representación en los dos sitios de exteriores fueron Lycosidae, Linyphiidae y Pholcidae, que se encontró exclusivamente en vivienda (Fig. 2 y 3).

En relación al número total de arañas, se encontró mayor abundancia en el campo con vegetación silvestre $(41 \%)$, seguido por la huerta $(36 \%)$ y menor abundancia en la vivienda (23\%). La mayor diversidad de familias se halló en campo con vegetación silvestre, 320 arañas pertenecientes a 17 familias. Se encontró una alta diversidad y abundancia en la huerta, 279 arañas pertenecientes a 14 familias. En la vivienda, encontramos que Pholcidae, Araneidae, Oecobiidae y Theridiidae estuvieron presentes durante todos los muestreos. Dos de estas familias solamente se encontraron en vivienda: Oecobidae y Pholcidae, esta última presentó una excepción en campo con vegetación silvestre en invierno. En otoño aparecieron uno o dos ejemplares de Corinnidae y Desidae, y en invierno Lycosidae y Ctenidae.algunas que generalmente se encuentran en el exterior. En verano se encontró la mayor abundancia de arañas (31\%), seguida de otoño (30\%), invierno (28\%) y finalmente la primavera presentó la menor abundancia general (11\%) (Fig. 4). Algunas familias presentaron una baja representación, como Gnaphosidae con tres ejemplares, uno en cada sitio del exterior en verano y uno en huerta en invierno y Clubionidae que apareció en otoño en los sitios del exterior y en invierno, un ejemplar en el campo con vegetación silvestre. El único representante de Titanoesidae fue encontrado en primavera en campo con vegetación silvestre. Durante todo el año la huerta y el campo con vegetación 


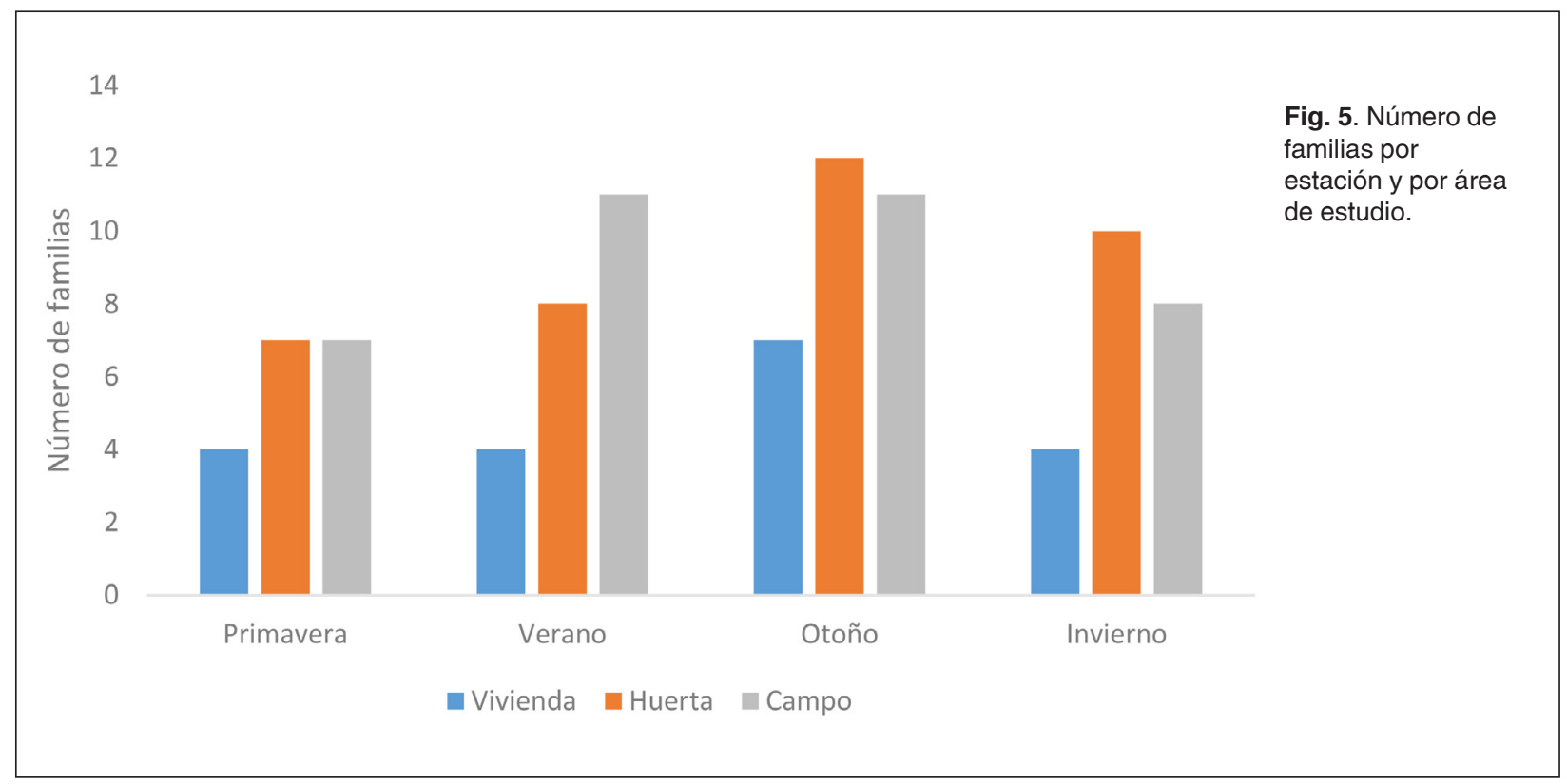

silvestre compartieron solamente las familias Linyphiidae y Lycosidae, En verano se compartieron mayor cantidad de familias en el huerta y campo con vegetación silvestre.

Se encontró un total de 20 arañas en actividad reproductiva pertenecientes a seis familias: Pholcidae, Linyphiidae, Lycosidae, Thomisidae, Araneidae y Theridiidae. El $60 \%$ de estas arañas pertenecen a la familia Pholcidae. Las estaciones con mayor actividad reproductiva fueron primavera y verano. En vivienda se encontraron en etapa reproductiva Araneidae en primavera, Pholcidae en primavera y verano, y Theridiidae en otoño. En huerta Lyniphidae en primavera y verano, Lycosidae en primavera y Thomisidae en verano. En campo con vegetación silvestre, solo un caso de Lycosidae en primavera.

\section{DISCUSIÓN}

La hipótesis planteada por los estudiantes, se corroboró con los resultados encontrados en el relevamiento. Las diferencias en el número de individuos y diversidad de familias entre los tres sitios pueden explicarse por diferencias en la cobertura vegetal del suelo. En vivienda fue reducida a plantas en macetas, correspondiendo al sitio con menor abundancia de arañas. En huerta, la cobertura vegetal consiste las plantas cultivadas en canteros paralelos, con hierbas asociadas, separados por corredores intercanteros de tierra de tamaño variable y sin cobertura vegetal. Este método agrícola crea un hábitat discontinuo, con islas (canteros) de vegetación. La cobertura en campo con especies silvestres es mayor que en la huerta, así como en diversidad de especies vegetales, que forman un hábitatcontinuo de pastos, hierbas y chircas. La mayor abundancia de arañas y la riqueza de familias encontradas en campo en la vegetación silvestre se debe a la mayor oferta de nichos que ofrece este tipo de hábitat. Ruiz (2017) encontró resultados similares comparando áreas verdes urbanas y áreas verdes perimetrales, en Córdoba, Argentina. Encontró mayor abundancia y riqueza de arañas en las áreas verdes externas, menos perturbadas que las urbanas. Resultados similares encontraron Torres et al. (2016) comparando la abundancia de la araña mirmecófaga Leprolochus birabeni en tres ambientes: nativo, de uso ganadero y de uso agrícola. Encontraron que, en relación con el ambiente nativo, la abundancia de arañas se redujo a un tercio en el ambiente agrícola, debido al uso de agroquímicos. Los autores encontraron, como en el presente trabajo, una relación inversa entre abundancia de arañas y grado de perturbación humana.

Respecto al relevamiento de familias de arañas, se encontraron familias comunes a los dos sitios exteriores en algunas épocas del año, lo que indica que la huerta, al no utilizar agroquímicos de manejo convencional, permitió encontrar una alta riqueza de familias. Este hallazgo esperable en el contexto de encierro, motivó una amplia reflexión sobre la conservación del medio ambiente libre de agrotóxicos y la importancia del mantenimiento de la biodiversidad. Los resultados encontrados fueron extraídos con rigurosidad y permitirán entonces su utilización en investigaciones futuras, como, por ejemplo, conocer 
los picos poblacionales en concordancia espaciotemporal con las potenciales presas (insectos-blanco). Por otra parte, la alta presencia de algunas arañas, como las pertenecientes a la familia Pholcidae (60\%) en las viviendas se debe a que una de sus especies más representativas Pholcus phalangioides está muy adaptada a vivir intramuros, fundamentalmente en ambientes húmedos, como baños y cocinas. Por otra parte, se observó la importancia de mantener zonas libres de intervención antrópica para favorecer reservorios de fauna nativa asociada. Los resultados de Torres et al. (2016) son similares a los encontrados por nosotros, respecto a la distribución de familias en los ambientes con presencia humana, pero no son extrapolables, debido a las condiciones en las que se realizaron los muestreos.

Se trabajaron las habilidades comunicacionales de los estudiantes para enfrentar a público académico específico, para las presentaciones en los eventos académicos ya mencionados. Pese a que el proyecto experimental fue el vehículo para desarrollar la tarea educativa, los resultados arrojaron datos interesantes que pueden ser utilizados por otros investigadores, debido al rigor científico con el que se realizó el relevamiento. Observamos una gran motivación que impulsó el trabajo en los estudiantes y en muchos casos, en los que había cierto grado de aversión hacia las arañas, no solamente se revirtió, sino que algunos de ellos criaron y cuidaron algunas arañas con dedicación. Además, los participantes desarrollaron tareas de divulgación in situ con otros compañeros de la Unidad penitenciaria.

El proceso educativo en el marco de la tarea de Educación Permanente, dentro de las tareas de Extensión de la UdelaR, se llevó a cabo en el ambiente social inherente a los estudiantes en la condición de personas privadas de libertad. Por lo tanto, las condiciones de estructura y utilización de material de laboratorio se vieron alteradas y limitadas. Esta situación creó una serie de desafíos que nos brindaron al conjunto de los participantes, la oportunidad de crear materiales alternativos que fuesen permitidos, superando las restricciones asociadas al contexto. La propuesta de integralidad ejecutada además de incluir el conocimiento técnico-científico, desarrolló los valores de conservación de nuestra fauna y el trabajo cooperativo en un ambiente responsable y respetuoso.

Los estudiantes aprendieron a aplicar un método sistemático para realizar el relevamiento a la luz de una hipótesis previamente elaborada. También aprendieron aspectos sobre la cría de animales. El mantenimiento de animales tiene un doble cometido, afinar habilidades y destrezas y por otra parte cultivar la paciencia, como contracultura del inmediatismo prevalente muchas veces en el medio. También cultivaron la solidaridad y el respeto necesario para el trabajo en grupo. Uno de los resultados de la experiencia educativa más importante fue el acercamiento de los estudiantes a un modelo animal no tradicional y temido por la mayoría de las personas.

Al adentrarnos en la temática en la que investigamos, observamos la curiosidad que despertó el comportamiento de las arañas y su importancia como depredadores de insectos-plaga. Además, encontraron otra importancia, como poder ser utilizados como bioindicadores de calidad ambiental. El conocimiento de la fauna que nos rodea y su valor como parte del patrimonio a conservar, también fue un resultado cualitativo de la experiencia educativa. El acercamiento a la "cocina" para la adquisición de conocimiento original resultó muy accesible y enriquecedora para todos los participantes.

Los resultados fueron discutidos y finalmente comunicados por los estudiantes durante el IV Congreso Uruguayo de Zoología celebrado en el CURE de Maldonado; y el V Congreso Uruguayo de Zoología celebrado en la Facultad de Ciencias, Montevideo (Bardier et al., 2016; Bardier et al., 2018). Se presentaron los resultados pedagógicos en el $\mathrm{X}$ Congreso Iberoamericano de Educación Científica, celebrado en el Palacio Municipal de la Intendencia de Montevideo (Viera et al. 2019).

La enseñanza de la investigación es un reto para todos los docentes universitarios. De acuerdo con Cabrera et al. (2015) este tipo de iniciativa se justifica para potenciar aptitudes y promover actitudes individuales, siendo los estudiantes proactivos en el involucramiento con la propuesta. De hecho, se trabajaron aspectos relacionados con trabajo en equipo, responsabilidad, autonomía, tolerancia a la frustración y fomentamos en todo momento el pensamiento crítico. Todo el trabajo se realizó con compromiso y responsabilidad, en un ambiente de respeto y diálogo entre personas privadas de libertad, funcionarios de la Unidad penitenciaria e investigadores y docentes de la Facultad de Ciencias y del Instituto de Investigaciones Biológicas Clemente Estable.

Impulsamos en este proyecto porque consideramos que la Universidad le pertenece a la sociedad y no solo debe responder a sus demandas como consignara Brovetto (1994). Este autor destaca que una actuación pertinente de la Universidad es cuando no se limita a actuar a demanda, sino que plantea proactivamente como colaborar con la educación en todos los niveles de la población, dentro y fuera del sistema educativo.

Para el cuerpo de docentes que colaboramos en la propuesta fue altamente removedor y desafiante el proceso. Nos enriquecemos no sólo como docentes, sino como seres humanos en evolución, derribando prejuicios y revalorando nuestro papel al servicio de los demás, en el intento de aportar a la construcción de una sociedad más justa y tolerante. Como reflexión final, nos parece que es necesario más emprendimientos y ofertas educativas de esta u otra modalidad para colaborar en la formación de las personas privadas de libertad, que no solo es un derecho inalienable, sino que puede prepararlos para su reinserción a nuestra sociedad. 


\section{AGRADECIMIENTOS}

A la Comisión de Educación Permanente de la Facultad de Ciencias que nos apoyó durante todo el proceso y facilitó los recursos para poder cumplir con la actividad. A la Unidad № 6 Punta de Rieles, en la figura de su Director, Sr. Luis Parodi, de quién también recibimos apoyo para cumplir nuestra tarea. Agradecemos a los revisores anónimos por sus sugerencias que mejoraron la calidad del trabajo.

\section{REFERENCIAS}

Aisenberg A., Toscano Gadea C. \& S. Ghione. 2011. Guía de Arácnidos del Uruguay. Ediciones de la Fuga.

Arocena R., Tomasino H., Rodríguez N., Sutz J., Alvarez Pedrocian E. \& A. Romano. 2011. Integralidad: tensiones y perspectivas. Cuadernos de Extensión (1). 107 pp.

Bardier G., Perlipe A., Costabel N., Parodi G., Rojas C. \& C. Viera. 2016. Relevamiento de arañas en la Unidad n 6 "Punta de Rieles" enmarcado en proyecto de extensión científico-educativo; comunicación preliminar. Actas del IV Congreso Uruguayo de Zoología: 79.

Bardier G., Mereles L., Costabel N., Vezzoli S., Perlipe A., López D., Parodi G., Rojas-Buffet C. \& C. Viera. 2018. Relevamiento de arañas en la unidad de internación de personas privadas de libertad № 6 "Punta de Rieles». Actas del IV Congreso Uruguayo de Zoología: 61.

Benamú M.A. 2007. Clave para la determinación de algunas familias de arañas (Araneae:Araneomorphae) del Uruguay. Boletín de la Sociedad Zoológica del Uruguay 2a época, 16: 1-19.

Brovetto J. 1994. Formar para lo desconocido: apuntes para la teoría y práctica de un modelo universitario de construcción. Serie Documentos de Trabajo (n5) de la Universidad de la República.

Cabrera C., Ferreño M. \& A. Davyt. 2015. Enseñar investigando. Diseño de cursos para la formación integral. $64 \mathrm{pp}$.

Costa F. \& M. Simó. 2014. Fenología de las arañas epígeas de una zona costera del sur de Uruguay: un estudio bianual con trampas de caída. Boletín de la Sociedad Zoológica de Uruguay (2a época), 23(1): 1-15.

Grismado C.J., Ramírez M.J. \& M. Izquierdo. 2014. Araneae: Taxonomía, diversidad y clave de identificación de familias. En: Roig-Juñent S., Claps L.E., Morrone J.J. (Eds.). Biodiversidad de Artrópodos Argentinos, vol. 3, pp. 55-93. INSUE-UNT/UADER, Argentina.

Laborda A, Montes de Oca L, Pérez-Miles F, Useta G. \& M. Simó. 2018. The spider fauna from Uruguay Riverislands: Undestanding its role in a biological corridor. Biodiversity Data Journal, 6: e27319.

Ruiz A.D. 2017. Efectos de la urbanización sobre las comunidades de arañas Araenidae Clerck 1757 (Arachnida:Araneae) presentes en los espacios verdes de la ciudad de Córdoba, Argentina. Tesis de Maestría de la Universidad Nacional de Córdoba, Argentina. 44 pp.

Simó M., Núñez M., Ojeda L., Laborda A. \& D. Queirolo. 2015. Knowing the biological linkage: spider composition and guilds in a hillrange of northern Uruguay. Boletín de La Sociedad Zoológica Del Uruguay, 24(2): 117-129.

Torres V.M., González-Reyes A.X., Rodriguez-Artigas S.M. \& J.A. Corronca. 2016. Effects of anthropogenic disturbance on the populations of Leprolochus birabeni (Araneae, Zodariidae) in the Chaco Seco northwestern Argentina. Iheringia. Série Zoologia (106). https://doi.org/ 10.1590/1678-4766e2016009

Viera C. 2011. Arácnidos de Uruguay: diversidad, ecología y comportamiento. Editorial Banda Oriental. 237 pp.

Viera C., Bardier G. \& C. Rojas-Buffet. 2019. Experiencia educativa en contexto de encierro: diversidad comparada de arañas. Enseñanza y Aprendizaje de las Ciencias en Debate, 4: 454.

Viera C. \& M.O. Gonzaga. 2017. Behaviour and Ecology of Spiders: Contributions from the Neotropical Region. Springer, $437 \mathrm{pp}$.

Fecha de Recepción: 12 de septiembre de 2020 Fecha de Aceptación: 17 de diciembre 2020 